\title{
STRUCTURAL CHARACTERIZATION OF A RECOMBINANT TN ANTIGEN-BINDING LECTIN FROM VATAIREA MACROCARPA
}

\author{
Sousa, B. L. ; Silva Filho, J. C. '; Pereira, R. I.1; Rocha, B. A. M. '; Delatorre, P.²; Be- \\ zerra, G. A. ${ }^{3}$; Nagano, C. S. ${ }^{1}$; Gruber, K.4; Cavada, B.S. ${ }^{1}$ \\ ${ }^{1}$ Department of Biochemistry and Molecular Biology, Federal University of Ceará, Fortaleza, Brazil \\ ${ }^{2}$ Department of Molecular Biology, Federal University of Paraíba, João Pessoa, Brazil \\ ${ }^{3}$ Institute of Molecular Biosciences, University of Graz, Graz, Austria \\ ${ }^{4}$ Department of Structural and Computational Biology, Max F. Perutz Laboratories, University of Vienna, Vienna, Austria
}

*brunolopesdesousa@gmail.com

Legume lectins represent the largest and most thoroughly studied lectin family, comprising a large group of homologous carbohydrate-binding proteins (Sharon \& Lis, 1990). A few galactose/N-acetylgalactosamine (Gal/GalNAc) binding lectins isolated from legume plants have proven to be useful as markers in cancer histochemistry. Structural analysis of Tn-binding lectins is of considerable interest for elucidating the mechanism of specific protein-carbohydrate recognition as well as for engineering novel binding activities in legume lectins. Here we present the crystal structures of a single-chain recombinant legume lectin from Vatairea macrocarpa (VML), recombinatly expressed in Escherichia coli, in complex with lactose, N-acetyl-galactosamine and Tn antigen, respectively. The structural data is supported by isothermal titration calorimetry (ITC) and small-angle X-ray scattering assays. Molecular docking simulations were also performed to analyze the binding of this lectin to $O$-mucins. These findings provide a complete structural characterization of this new Tn-binding protein and strongly suggest recombinant VML as a new tool for cancer research. 